\title{
SYSTOLIC VOLUME OF HYPERBOLIC MANIFOLDS AND CONNECTED SUMS OF MANIFOLDS
}

\author{
STÉPHANE SABOURAU
}

\begin{abstract}
The systolic volume of a closed $n$-manifold $M$ is defined as the optimal constant $\sigma(M)$ satisfying the inequality $\operatorname{vol}(M) \geq \sigma(M) \operatorname{sys}(M)^{n}$ between the volume and the systole of every metric on $M$. First, we show that the systolic volume of connected sums of closed oriented essential manifolds is unbounded. Then, we prove that the systolic volume of every sequence of closed hyperbolic (three-dimensional) manifolds is also unbounded. These results generalize systolic inequalities on surfaces in two different directions.
\end{abstract}

\section{INTRODUCTION}

Consider a nonsimply connected closed $n$-manifold $M$ endowed with a Riemannian metric $g$. The systole of $(M, g)$, denoted sys $(M, g)$, is defined as the length of the shortest noncontractible loop in $M$. Define the systolic volume of $M$ as

$$
\sigma(M)=\inf _{g} \frac{\operatorname{vol}(M, g)}{\operatorname{sys}(M, g)^{n}}
$$

where $g$ runs over the space of all metrics on $M$.

The systolic volume is a homotopy invariant, $c f$. [Ba93]. Furthermore, in a large number of cases, the systolic volume of a manifold depends only on its homology class in the corresponding Eilenberg-MacLane space ( $c f$. [Ba04] and $[\mathrm{Ba} 06]$ for a more precise statement).

A closed oriented $n$-manifold $M$ is said to be essential if there is a map $\Phi: M \rightarrow K(\pi, 1)$ to some $K(\pi, 1)$-space such that $\Phi_{*}[M]$ is nonzero in $H_{n}(\pi ; \mathbb{Z})$. Note that closed oriented aspherical manifolds are essential. Furthermore, the connected sum of an essential manifold with any closed oriented manifold is essential.

M. Gromov [Gr83] proved that the systolic volume of essential manifolds is bounded away from zero ( $c f$. $[\mathrm{Gu}]$ for a recent extension). More precisely, there exists a positive constant $C_{n}$ such that every essential $n$-manifold $M$ satisfies

$$
\sigma(M) \geq C_{n} .
$$

Conversely, I. Babenko [Ba93] showed that a closed oriented manifold with positive systolic volume is essential.

Key words and phrases. systole, systolic volume, connected sums, hyperbolic manifolds. 
The systolic inequality (1.2) can be improved by taking into account the topology of the manifold. For instance, M. Gromov [Gr83, 6.4.C' \& 6.4.D'], [Gr96, 3.C.3] showed that the systolic volume of a closed manifold $M$ is bounded from below in terms of its simplicial volume $\|M\|$ and its simplicial height $h(M)$ (cf. Section 2 for a definition). Specifically, there exist two positive constants $C_{n}$ and $C_{n}^{\prime}$ depending only on $n$ such that every closed $n$-manifold $M$ satisfies

$$
\begin{gathered}
\sigma(M) \geq C_{n} \frac{\|M\|}{\log ^{n}(1+\|M\|)}, \\
\sigma(M) \geq C_{n} \frac{h(M)}{\exp \left(C_{n}^{\prime} \sqrt{\log h(M)}\right)} .
\end{gathered}
$$

When $M$ is aspherical, $n \neq 3$, the author [Sa06] showed that the simplicial volume can be replaced by the minimal entropy in (1.3).

In the two-dimensional case, the inequality (1.3), improved in [KS05], yields a lower bound on the systolic volume of surfaces (an asymptotic lower bound for general 2-complexes can be found in [RS]). The examples of hyperbolic surfaces with large systoles obtained in [BS94] (cf. [KSV05] for other examples and generalizations in higher dimension) provide a similar upper bound on the systolic volume. Specifically, there exists an explicit positive constant $c$ such that every closed orientable surface $\Sigma_{k}$ of genus $k$ satisfies

$$
\pi \frac{k}{(\log k)^{2}} \lesssim \sigma\left(\Sigma_{k}\right) \lesssim c \frac{k}{(\log k)^{2}} .
$$

The surface $\Sigma_{k}$ can be described as a connected sum of $k$ tori or as a surface admitting a hyperbolic metric (of area $4 \pi(k-1)$ ). In higher dimension, no manifold satisfies these two features, but we can separately study the systolic volume of the manifolds satisfying one or the other.

The first result we obtain deals with connected sums.

Theorem A. Let $M$ be a closed oriented essential n-manifold. Then, the systolic volume of the connected sums $\#_{k} M=M \# \ldots \# M$ of $k$ copies of $M$ is unbounded. More precisely,

$$
\sigma\left(\#_{k} M\right) \geq C_{n} \frac{k}{\exp \left(C_{n}^{\prime} \sqrt{\log k}\right)}
$$

where $C_{n}$ and $C_{n}^{\prime}$ are two positive constants depending only on $n$.

We can also replace $\#_{k} M$ by the connected sum $M_{1} \# \cdots \# M_{k}$ of $k$ closed oriented essential $n$-manifolds, $c f$. Theorem 3.1 for a slightly more general version. If $M$ is non-essential then $\#_{k} M$ is also non-essential and its systolic volume vanishes, $c f$. [Ba93].

Theorem A provides a partial answer to a question raised in [Gr96, p. 330] and $\left[\mathrm{BB} 05, Q_{1}\right]$ asking for the asymptotic behaviour of $\sigma\left(\#_{k} M\right)$. In [BB05], 
I. Babenko and F. Balacheff show that, for every closed $n$-manifold $M$, there exists a constant $C(M)$ depending only on the topology of $M$ such that

$$
\sigma\left(\#_{k} M\right) \leq C(M) \frac{k \sqrt{\log \log k}}{\sqrt{\log k}} .
$$

This estimate provides a sublinear upper bound on $\sigma\left(\#_{k} M\right)$. They also conjecture that for every closed essential $n$-manifold $M$

$$
\sigma\left(\#_{k} M\right) \sim \frac{k}{(\log k)^{n}} .
$$

Example 1.1. The systolic volume of $k$ copies of $n$-tori goes to infinity with $k$, i.e., $\sigma\left(\mathbb{T}^{n} \# \cdots \# \mathbb{T}^{n}\right) \rightarrow \infty$. This result does not follow from the inequality (1.3) since the simplicial volume $\left\|\mathbb{T}^{n}\right\|$ of the torus is zero for $n \geq 3$, cf. [Gr81]. When $n=2$, this result follows from the estimate (1.5).

The second result we obtain deals with hyperbolic manifolds.

Theorem B. Let $\left\{M_{i}\right\}$ be a sequence of infinitely many, non-homeomorphic, closed hyperbolic n-manifolds. Then, the systolic volume of the $M_{i}$ 's is unbounded, that is

$$
\lim _{i \rightarrow \infty} \sigma\left(M_{i}\right)=\infty
$$

This result is already known for $n=2$ and $n \geq 4$. In the two-dimensional case, it follows from the asymptotic bound (1.5). When $n \geq 4$, H. C. Wang's theorem [Wa72] (see [BGLM02] for a quantitative version) asserts that there are only finitely many closed hyperbolic $n$-manifolds with bounded simplicial volume (recall that the simplicial volume is proportional to the hyperbolic volume, cf. [Th97], [Gr81]). Combined with the inequality (1.3), this implies Theorem B for $n \geq 4$. Since the conclusion of H. C. Wang's theorem does not hold for $n=3$, the three-dimensional case requires other arguments.

This paper is organized as follows. In Section 2, we introduce the notions of relative systoles and simplicial heights. We also recall a very general systolic inequality due to M. Gromov, which relates the systolic volume of a manifold to its simplicial height, generalizing the inequality (1.4). This systolic inequality plays a central role in the proofs of Theorems A and B. In Section 3, we introduce some constructions and notations about the connected sums of manifolds. In Section 4, we bound from below the simplicial height of the connected sum $\#_{k} M$ by $k$, which leads to Theorem A. The proof of Theorem B proceeds as follows. In [So98], T. Soma proved a finiteness result for closed orientable hyperbolic manifolds dominated by the same manifold. Recently, G. Besson, G. Courtois and S. Gallot [BCG05] found another proof of this result based on the deformation of representations in the isometry group of the hyperbolic space. A straightforward generalization of their arguments shows that T. Soma's theorem applies to cycles, 
$c f$. Sections 5 and 6 . We deduce then that the simplicial height of a sequence of closed orientable hyperbolic manifolds is unbounded, which leads to Theorem B.

Acknowledgement. Part of this work was completed during the program "Spaces of negative curvature" at the Centre Interfacultaire Bernoulli, where the author tried to construct a counterexample to Theorem B, see Example 6.3. The author would like to thank the Centre for its hospitality and the participants, including F. Balacheff, C. Croke, H. Parlier and A. Reid, for several helpful discussions.

\section{Systolic Volume AND Simplicial Height}

Let $\pi$ be a discrete group. A geometric cycle representing a homology class $\alpha$ in $H_{n}(\pi ; \mathbb{Z})$ is a map $\Psi: X \longrightarrow K(\pi, 1)$ from a closed oriented $n$ dimensional Riemannian pseudomanifold $(X, g)$ ( $c f$. [Sp66] for a definition) to a $K(\pi, 1)$-space such that $\Psi_{*}[X]=\alpha$ where $[X]$ is the fundamental class of $X$. Denote by $\psi: \pi_{1}(X) \rightarrow \pi$ the homomorphism induced by $\Psi$ and by $\psi_{\sharp}$ the map induced by $\psi$ between the conjugacy classes. The $\psi$-systole of $(X, g)$, denoted $\operatorname{sys}_{\psi}(X, g)$, is defined as the length of the shortest loop of $X$ whose image by $\psi_{\sharp}$ is nontrivial. The $\psi$-systolic volume of $(X, g)$ is defined as

$$
\sigma_{\psi}(X, g)=\frac{\operatorname{vol}(X, g)}{\operatorname{sys}_{\psi}(X, g)^{n}} .
$$

Here, the volume of an $n$-dimensional Riemannian polyhedron $(X, g)$ is defined as the sum of the $n$-volumes of all the $n$-simplices of $X$. Thus, the $n$-volume agrees with the $n$-dimensional Hausdorff measure. We clearly have

$$
\sigma_{\psi}(X) \leq \sigma(X) .
$$

The systolic volume of a homology class $\alpha \in H_{n}(\pi ; \mathbb{Z})$ is defined as

$$
\sigma(\alpha)=\inf _{X} \sigma_{\psi}(X, g),
$$

where $X=(X, \Psi, g)$ runs over all the geometric cycles representing $\alpha$.

M. Gromov showed in $[\mathrm{Gr} 83, \S 6]$ that there exists a positive constant $c_{n}$ such that every nontrivial homology class $\alpha \in H_{n}(\pi ; \mathbb{Z})$ satisfies

$$
\sigma(\alpha) \geq c_{n}
$$

By definition, if $M$ is a closed oriented essential manifold, there exists a map $\Phi: M \rightarrow K(\pi, 1)$ such that $\Phi_{*}[M]$ is nonzero in $H_{n}(\pi, \mathbb{Z})$. Thus,

$$
\sigma\left(\Phi_{*}[M]\right) \leq \sigma(M) .
$$

In particular, every closed essential $n$-manifold $M$ satisfies $\sigma(M) \geq c_{n}$.

Example 2.1. Given a closed Riemannian manifold $M$, the homology modulo torsion, denoted $H_{*}(M ; \mathbb{Z})_{\mathbb{R}}$, is the image of $H_{*}(M ; \mathbb{Z})$ in $H_{*}(M ; \mathbb{R})$. 
Suppose there exist $n$ cohomology classes in $H^{1}(M ; \mathbb{R})$ whose cup product is nonzero in $H^{n}(M ; \mathbb{R})$. Then, the natural map

$$
\Phi: M \rightarrow K\left(H_{1}(M ; \mathbb{Z})_{\mathbb{R}}, 1\right) \simeq H_{1}(M ; \mathbb{R}) / H_{1}(M ; \mathbb{Z})_{\mathbb{R}}
$$

to the Jacobi variety takes the fundamental class of $M$ to a nonzero class in homology. The inequality (2.4) yields a systolic inequality for the homology systole (modulo torsion) defined as $\operatorname{sys}_{H}(M)=\operatorname{sys}_{\phi}(M)$ where $\phi: \pi_{1}(M) \rightarrow$ $H_{1}(M ; \mathbb{Z})_{\mathbb{R}}$ is the natural homomorphism. Namely,

$$
\operatorname{sys}_{H}(M)^{n} \leq c_{n} \operatorname{vol}(M) .
$$

Since the stable systole, $c f$. [CK03] for a definition, satisfies $\operatorname{stsys}(M) \leq$ $\operatorname{sys}_{H}(M)$, this leads to the inequality,

$$
\operatorname{stsys}(M)^{n} \leq c_{n} \operatorname{vol}(M)
$$

where the constant $c_{n}$ depends only on $n$ (in particular, it is independent of the (first) Betti number of $M$ ). Compare with [BK03], [IK04] where more general systolic inequalities have been established for the stable systole but with a constant depending on the Betti numbers of $M$. See also [BK03], [BK04], [IK04], [BCIK06], [KR] for examples of sharp systolic inequalities for the stable and conformal systoles along with a description of the extremal metrics and [CK03] for a general account on the subject.

By using regularization techniques on geometric cycles, M. Gromov also showed that the lower bound (2.4) can be improved by taking into account the topology of the representatives of the homology class $\alpha$ as follows.

Definition 2.2 ([Gr96, 3.C.3]). The simplicial height of a homology class $\alpha \in$ $H_{n}(\pi ; \mathbb{Z})$, denoted $h(\alpha)$, is the minimal possible number of simplices of an oriented pseudomanifold $X$ which admits a map $\Psi: X \rightarrow K(\pi, 1)$ to some $K(\pi, 1)$-space such that $\Psi_{*}[X]=\alpha$ where $\Psi_{*}: H_{n}(X ; \mathbb{Z}) \rightarrow H_{n}(\pi ; \mathbb{Z})$ is the homomorphism induced by $\Psi$. The simplicial height of a closed orientable manifold $M$, denoted $h(M)$, is defined as the simplicial height of the image of its fundamental classs under the homomorphism $\Phi_{*}: H_{n}(M ; \mathbb{Z}) \rightarrow$ $H_{n}\left(\pi_{1}(M) ; \mathbb{Z}\right)$ induced by its classifying map.

The following estimate, to compare with (1.4), improves the bound (2.4).

Theorem 2.3 ([Gr83, 6.4.C'] \& [Gr96, 3.C.3]). There exist two positive constants $C_{n}$ and $C_{n}^{\prime}$ depending only on $n$ such that every nontrivial homology class $\alpha \in H_{n}(\pi ; \mathbb{Z})$ satisfies

$$
\sigma(\alpha) \geq C_{n} \frac{h(\alpha)}{\exp \left(C_{n}^{\prime} \sqrt{\log h(\alpha)}\right)} .
$$

\section{Relative Systolic Volume of CONNECTEd Sums of MANifoldS}

Fix $n \geq 3$. Let $\Phi_{i}: M_{i} \longrightarrow K_{i}=K\left(\pi_{i}, 1\right), 1 \leq i \leq k$, be maps between closed oriented $n$-dimensional manifolds $M_{i}$ to aspherical spaces $K_{i}$ with fundamental group $\pi_{i}$. The maps $\Phi_{i}$ induce homomorphisms $\phi_{i}: \pi_{1}\left(M_{i}\right) \longrightarrow \pi_{i}$ 
between the fundamental groups and homomorphisms $\Phi_{i, *}: H_{n}\left(M_{i} ; \mathbb{Z}\right) \longrightarrow$ $H_{n}\left(\pi_{i} ; \mathbb{Z}\right)$ between the $n$-th homology groups. Note that the $K_{i}$ are $K\left(\pi_{i}, 1\right)$ spaces.

Let $M$ be the connected sum of $M_{1}, M_{2}, \ldots, M_{k}$, that is

$$
M=M_{1} \# M_{2} \# \ldots \# M_{k},
$$

and $K$ be the bouquet of the aspherical spaces $K_{1}, K_{2}, \ldots, K_{k}$, that is

$$
K=K_{1} \vee K_{2} \vee \cdots \vee K_{k} .
$$

The space $K$ is also aspherical. Since $n \geq 3$, the fundamental groups of $M$ and $K$ are respectively isomorphic to the following free products

$$
\begin{gathered}
\pi_{1}(M)=\pi_{1}\left(M_{1} \# \ldots \# M_{k}\right) \simeq \pi_{1}\left(M_{1}\right) * \cdots * \pi_{1}\left(M_{k}\right) \\
\pi_{1}(K)=\pi_{1}\left(K_{1} \vee \cdots \vee K_{k}\right) \simeq \pi_{1} * \cdots * \pi_{k} .
\end{gathered}
$$

We will denote by $\pi$ the fundamental group of $K$. Thus, $K$ is a $K(\pi, 1)$ space with $\pi \simeq \pi_{1} * \pi_{2} * \cdots * \pi_{k}$.

Define a map $\Phi: M \longrightarrow K$ as follows

$$
\Phi: M_{1} \# \ldots \# M_{k} \longrightarrow M_{1} \vee \cdots \vee M_{k} \longrightarrow K_{1} \vee \cdots \vee K_{k} .
$$

The map $\Phi$ first contracts the attaching spheres of $M_{1} \# \ldots \# M_{k}$ into a point, then takes each component $M_{i}$ of the bouquet $M_{1} \vee \cdots \vee M_{k}$ to the corresponding component $K_{i}$ of $K_{1} \vee \cdots \vee K_{k}$ through $\Phi_{i}$. The homomorphism $\phi: \pi_{1}(M) \longrightarrow \pi$ induced by $\Phi$ between the fundamental groups agrees with the homomorphism $\phi_{1} * \cdots * \phi_{k}$ from $\pi_{1}\left(M_{1}\right) * \cdots * \pi_{1}\left(M_{k}\right)$ to $\pi_{1} * \cdots * \pi_{k}$ which takes every element $x$ of $\pi_{1}\left(M_{i}\right)$ to $\phi_{i}(x)$ in $\pi_{1} * \cdots * \pi_{k}$.

Using the previous notations, we can state the following result, whose proof occupies the next section.

Theorem 3.1. Let $\Phi_{i}: M_{i} \longrightarrow K_{i}, 1 \leq i \leq k$, be maps as above. Suppose that, for every $i$, the class $\Phi_{i *}\left[M_{i}\right]$ is nontrivial in $H_{n}\left(\pi_{i} ; \mathbb{Z}\right)$. Then,

$$
\sigma_{\phi}\left(M_{1} \# \ldots \# M_{k}\right) \geq C_{n} \frac{k}{\exp \left(C_{n}^{\prime} \sqrt{\log k}\right)},
$$

where $C_{n}$ and $C_{n}^{\prime}$ are two positive constants depending only on $n$.

Remark 3.2. The assumptions of Theorem 3.1 mean exactly that the manifolds $M_{i}$ are essential. Using the inequality (2.2), Theorem 3.1 yields Theorem A.

Remark 3.3. It should be noted, $c f$. [Ba95, BB05, Ba06], that adding a non-essential manifold to an essential manifold does not change the systolic volume. More precisely, let $M$ and $N$ be two closed oriented $n$-manifolds with $M$ essential and $N$ non-essential then $\sigma(M \# N)=\sigma(M)$. 


\section{Proof of Theorem A}

Let us prove Theorem 3.1, which implies Theorem A.

Using the notations of the previous section, the $n$-th homology group $H_{n}(\pi ; \mathbb{Z})$ of the bouquet $K$ of the $K\left(\pi_{i} ; \mathbb{Z}\right)$-spaces $K_{i}$ is given by

$$
H_{n}(\pi ; \mathbb{Z}) \simeq \bigoplus_{i=1}^{k} H_{n}\left(\pi_{i} ; \mathbb{Z}\right) .
$$

By construction, the map $\Phi, c f$. (3.1), takes the fundamental class of $M$ to the sum of the $\Phi_{i *}\left[M_{i}\right]$, that is

$$
\Phi_{*}[M]=\Phi_{1 *}\left[M_{1}\right] \oplus \cdots \oplus \Phi_{k *}\left[M_{k}\right] \in H_{n}(\pi ; \mathbb{Z}) .
$$

Let us show that the simplicial height of $\Phi_{*}[M]$ is bounded from below by $k$. Consider a map $\Psi: X \longrightarrow K$ from an oriented $n$-dimensional pseudomanifold $X$ to $K$ such that

$$
\Psi_{*}[X]=\Phi_{*}[M],
$$

where $\Psi_{*}: H_{n}(X ; \mathbb{Z}) \rightarrow H_{n}(\pi ; \mathbb{Z})$ is the homomorphism beween the $n$-th homology groups induced by $\Psi$. Denote by $\psi: \pi_{1}(X) \longrightarrow \pi$ the homomorphism between the fundamental groups induced by $\Psi$.

By Grushko's theorem [Ma77, $\S 7.6]$, the image $G=\psi\left(\pi_{1}(X)\right)$ of $\psi$ decomposes into a free product of subgroups

$$
G=F_{p} * H_{1} * \cdots * H_{r} \subset \pi
$$

such that $F_{p}$ is free of rank $p$, while every $H_{i}$ is nontrivial, non isomorphic to $\mathbb{Z}$ and freely indecomposable. Furthermore, given another decomposition of this sort, say $G=F_{q} * H_{1}^{\prime} * \cdots * H_{s}^{\prime}$, one necessarily has $p=q, r=s$ and, after reordering, $H_{i}^{\prime}$ is conjugate to $H_{i}$.

STEP 1. Our first step amounts to drop the free factor $F_{p}$ in the Grushko decomposition of $G$.

The homomorphism $\psi: \pi_{1}(X) \rightarrow \pi$ factors through the following commutative diagram

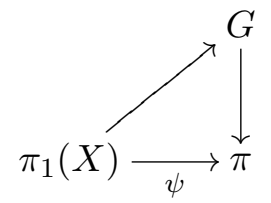

where $\pi_{1}(X) \rightarrow G$ is the epimorphism induced by $\psi$ and $G \hookrightarrow \pi$ is the monomorphism given by the inclusion. Therefore, the map $\Psi$ factors, up to homotopy, through the classifying maps induced by the above epimorphism and monomorphism, according to the following commutative (up to homotopy) diagram 


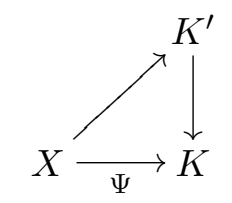

where $K^{\prime}$ is a $K(G, 1)$-space.

The composite $\Psi^{\prime}: X \rightarrow K^{\prime} \rightarrow K$ of these classifying maps and the map $\Psi$ take $[X]$ to the same class, that is

$$
\Psi_{*}[X]=\Psi_{*}^{\prime}[X] .
$$

Since $G=F_{p} * H$ with $H=H_{1} * \cdots * H_{r}$, the Eilenberg-MacLane space $K^{\prime}=K(G, 1)$ can be realized (up to homotopy equivalence) as $K^{\prime}=K^{\prime \prime} \vee$ $\bigvee_{i=1}^{p} S^{1}$, where $K^{\prime \prime}$ is a $K(H, 1)$-space. The projection of $K^{\prime}$ to $K^{\prime \prime}$ defines a map $K^{\prime} \rightarrow K^{\prime \prime}$. Consider the natural composite

$$
\Theta: X \rightarrow K^{\prime} \rightarrow K^{\prime \prime} \rightarrow K
$$

where the map $K^{\prime \prime} \rightarrow K$ is the classifying map induced by the inclusion homomorphism $H \hookrightarrow G$. By construction, we have $\Theta_{*}[X]=\Psi_{*}^{\prime}[X]$. From (4.1) and (4.2), this relation can be written as

$$
\Theta_{*}[X]=\Phi_{*}[M] .
$$

Furthermore, the image of the homomorphism $\theta: \pi_{1}(X) \rightarrow G$ induced by $\Theta$ agrees with $H$, that is $\theta\left(\pi_{1}(X)\right)=H$.

This shows that, for our purpose, we can drop the free factor in Grushko's decomposition.

STEP 2. Consider now another natural composite

$$
\Theta_{j}: X \stackrel{\Theta}{\rightarrow} K \rightarrow K_{j},
$$

where the second map $K \rightarrow K_{j}$ is the projection of $K$ to $K_{j}$. Note that the homomorphism $p_{j}: \pi \rightarrow \pi_{j}$ induced by $K \rightarrow K_{j}$ is trivial on each free factor $\pi_{i}$ of $\pi$ with $i \neq j$.

Since $\Theta_{*}[X]=\Phi_{*}[M]$, the homomorphism between the $n$-th homology groups induced by $\Theta_{j}$ takes $[X]$ to the nontrivial class $\Phi_{j *}\left[M_{j}\right]$. Therefore, the homomorphism $\theta_{j}: \pi_{1}(X) \stackrel{\theta}{\rightarrow} H \stackrel{p_{j}}{\rightarrow} \pi_{j}$ induced by $\Theta_{j}$ is nontrivial.

STEP 3. By Kurosh's theorem [Ma77, §7.5], the subgroup $H_{i}$ of $\pi=$ $\pi_{1} * \cdots * \pi_{k}$ is conjugate in $\pi$ to a subgroup of $\pi_{j}$ with $j=\tau(i)$. This property fails if $H_{i}$ is a nontrivial free subgroup of $\pi$. Indeed, one can define a homomorphism from $F_{p}$ to $\pi$ by taking each element of a natural basis of $F_{p}$ to any element of $\pi$. This justifies our first step.

Thus, if $j$ is different from $\tau(i)$, then $p_{j}\left(H_{i}\right)$ is trivial. Therefore, the map $\tau$ from $\{1, \cdots, r\}$ to $\{1, \cdots, k\}$ is surjective, otherwise there would be a trivial homomorphism $\theta_{j}: \pi_{1}(X) \rightarrow H \rightarrow \pi_{j}$ with $j \notin \operatorname{Im} \tau$, contradicting Step 2. Thus, we have $r \geq k$. 
STEP 4. We can now conclude the proof of Theorem 3.1, which implies Theorem A.

By Grushko's theorem ( $c f$. [Ma77, $\S 7.6])$, we have $\operatorname{rank} G \geq r$. Thus,

$$
\operatorname{rank} \pi_{1}(X) \geq \operatorname{rank} G \geq r \geq k .
$$

Since the number of 1 -simplices in $X$ bounds from above the rank of $\pi_{1}(X)$, we obtain

$$
h\left(\Phi_{*}[M]\right)=h\left(\Psi_{*}[X]\right) \geq k,
$$

where the simplicial height $h$ is defined in Section 2 .

Using (2.6), we deduce

$$
\sigma_{\phi}\left(M_{1} \# \ldots \# M_{k}\right) \geq C_{n} \frac{k}{\exp \left(C_{n}^{\prime} \sqrt{\log k}\right)} .
$$

Hence Theorem 3.1.

\section{Deformations of Representations in Hyperbolic SPACES}

Let $P$ be a finite simplicial $n$-complex and $\tilde{P}$ be its universal cover (on which $\pi_{1}(P)$ acts by deck transformations). Let $\rho: \pi_{1}(P) \rightarrow \operatorname{Isom}\left(\mathbb{H}^{n}\right)$ be a representation from $\pi_{1}(P)$ to the isometry group of the hyperbolic $n$ space $\mathbb{H}^{n}$. Choose a piecewise smooth $\rho$-equivariant map $f: \tilde{P} \rightarrow \mathbb{H}^{n}$. The pullback $f^{*}(\omega)$ of the volume form $\omega$ on $\mathbb{H}^{n}$ descends to a simplicial form on $P$, still denoted by $f^{*}(\omega)$. Define the $\rho$-volume of an $n$-cycle $c$ of $P$ as follows:

$$
\operatorname{vol}(\rho, c)=\int_{c} f^{*}(\omega)
$$

The $\rho$-volume of an $n$-cycle is independent of the choice of $f$. Indeed, two piecewise smooth $\rho$-equivariant maps $f_{0}$ and $f_{1}$ from $\tilde{P}$ to $\mathbb{H}^{n}$ are $\rho$ equivariantly homotopic. Thus, the pullbacks $f_{t}^{*}(\omega)$ of the volume form $\omega$ on $\mathbb{H}^{n}$ by this $\rho$-equivariant homotopy $f_{t}$ passe to the quotient on $P$. Their quotients on $P$ define a homotopy between $f_{0}^{*}(\omega)$ and $f_{1}^{*}(\omega)$ (viewed as simplicial forms on $P$ ). Thus, these two forms lie in the same cohomology class through the De Rham isomorphism for simplicial complexes, cf. [Sw75]. Therefore, the $\rho$-volume $\operatorname{vol}(\rho, c)$ of an $n$-cycle $c$ of $P$ is well-defined (see [Du99] for further details).

The following proposition generalizes to finite simplicial complexes a result established in [Re96] for closed oriented 3-manifolds and in [BCG05, §6] for closed oriented $n$-manifolds. Note that if $c$ is not a cycle but only a chain, the $\rho$-volume of $c$ depends on the $\rho$-equivariant map $f$ and the conclusion of the proposition fails.

Proposition 5.1. Let $c$ be an n-cycle of a finite simplicial $n$-complex $P$. There exists a finite set $\Lambda \subset \mathbb{R}$ such that the $\rho$-volume of $c$ lies in $\Lambda$ for every representation $\rho: \pi_{1}(P) \rightarrow \operatorname{Isom}\left(\mathbb{H}^{n}\right)$. 
Proof. We adapt the arguments of [BCG05, §6], developing only the points that differ from that reference.

Since the space of representations $\mathcal{R}$ from $\pi_{1}(P)$ to $\operatorname{Isom}\left(\mathbb{H}^{n}\right)$ is an algebraic variety, it has finitely many path-connected components. For our purpose, it is therefore enough to show that $\operatorname{vol}(., c)$ is constant on each of these path-connected components.

Let $\rho_{t}: \pi_{1}(P) \rightarrow \operatorname{Isom}\left(\mathbb{H}^{n}\right), t \in \mathbb{R}$, be a $C^{1}$-family of representations. By [BCG05, Lemme 6.2], there exists a $C^{1}$-family of nondegenerate $\rho_{t^{-}}$ equivariant affine maps $f_{t}: \tilde{P} \rightarrow \mathbb{H}^{n}$ defined for $|t|$ small enough. Here, a map from a simplicial complex to $\mathbb{H}^{n}$ is said to be affine if it is affine along the geodesic arcs of every (Euclidean) simplex. Such a map is said to be nondegenerate if the image of every simplex is a nondegenerate (geodesic) simplex of $\mathbb{H}^{n}$. Strictly speaking, this result has been stated in [BCG05] for closed manifolds, but the proof extends to finite polyhedra.

Fix an orientation on every $n$-simplex of $P$. Let $c=\sum_{i \in I} k_{i} s_{i}$ be an $n$-cycle of $P$, where the $s_{i}$ are $n$-simplices of $P$. Let $F$ be an oriented 2codimensional simplex of $P$. An $n$-simplex $s$ of $P$ with $F$ as an $(n-2)$-face has exactly two $(n-1)$-faces meeting along $F$. The barycenters of these two faces can be joined by a segment of $s$. The link around $F$ is defined as the union of all these segments. Thus, the link around $F$ is a (possibly multiconnected) graph $\Gamma$ whose edges are in one-to-one correspondence with the $n$-simplices of $P$ with $F$ as a face. The orientation of $F$ along with the orientations of the $n$-simplices of $P$ induce an orientation on the edges of $\Gamma$.

Let $s_{j}, j \in J_{F} \subset I$, be the $n$-simplices $s_{i}$ of $c$ with $F$ as a face. Denote by $e_{j}$ the (oriented) edges of $\Gamma$ corresponding to the $n$-simplices $s_{j}$ of $P$. Since $c$ is a cycle, the 1-chain $e=\sum_{j \in J_{F}} k_{j} e_{j}$ of $\Gamma$ is a cycle too.

The geodesic simplex $F_{t}:=f_{t}(F)$ of $\mathbb{H}^{n}$ is endowed with the orientation of $F$. Since $\mathbb{H}^{n}$ is an oriented manifold, the link around $F_{t}$ is a circle $\mathcal{C}_{t}$ endowed with the induced orientation. The map $f_{t}$ induces a map $\varphi_{t}: \Gamma \rightarrow \mathcal{C}_{t}$ between the links. The homomorphism $\varphi_{t *}: H_{1}(\Gamma, \mathbb{Z}) \rightarrow H_{1}\left(\mathcal{C}_{t}, \mathbb{Z}\right) \simeq \mathbb{Z}$ induced by $\varphi_{t}$ takes the homology class of the 1-cycle $e$ to an integral multiple $k$ of the fundamental class of $\mathcal{C}_{t}$. Since $f_{t}$ is nondegenerate for $|t|$ small enough, the integer $k$, also noted $\operatorname{deg}_{F} f_{t}$, does not depend on $t$ for $|t|$ small enough.

Given an $n$-simplex $s$ of $P$ with $F$ as a face, let $\theta(t ; F, s)$ be the nonnegative hyperbolic dihedral angle of $f_{t}(s)$ at $F_{t}$. Define $\epsilon_{i}$ as 1 if the restriction of $f_{t}$ to the $n$-simplex $s_{i}$ (which is an affine map) preserves the orientation and as -1 otherwise. As previously, since $f_{t}$ is nondegenerate for $|t|$ small enough, the value $\epsilon_{i}$ does not depend on $t$ for $|t|$ small enough.

By adapting the proofs of the lemmas 6.3 and 6.4 in [BCG05], one can show the following

Lemma 5.2 (Compare with [BCG05, Lemmes 6.3 \& 6.4]). We have

$$
2 \pi \operatorname{deg}_{F} f_{t}= \pm \sum_{j \in J_{F}} \epsilon_{j} k_{j} \theta\left(t ; F, s_{j}\right) .
$$


In particular,

$$
\frac{d}{d t}\left(\sum_{j \in J_{F}} \epsilon_{j} k_{j} \theta\left(t ; F, s_{j}\right)\right)=0
$$

for $|t|$ small enough.

We can now conclude as in [BCG05, §6]. By definition,

$$
\begin{aligned}
\operatorname{vol}\left(\rho_{t}, c\right) & =\int_{c=\sum k_{i} s_{i}} f_{t}^{*}(\omega) \\
& =\sum_{i \in I} \epsilon_{i} k_{i} \operatorname{vol}\left(f_{t}\left(s_{i}\right)\right) .
\end{aligned}
$$

Its derivative can be expressed using Schläfli's differential formula, $c f$. [Mi94],

$$
\begin{aligned}
\frac{d}{d t} \operatorname{vol}\left(\rho_{t}, c\right) & =\sum_{i \in I} \epsilon_{i} k_{i}\left[-\frac{1}{n-1} \sum_{F \subset s_{i}} \frac{d}{d t}\left(\theta\left(t ; F, s_{i}\right)\right) \operatorname{vol}_{n-2}\left(F_{t}\right)\right] \\
& =-\frac{1}{n-1} \sum_{F \subset c} \frac{d}{d t}\left[\sum_{j \in J_{F}} \epsilon_{j} k_{j} \theta\left(t ; F, s_{j}\right)\right] \operatorname{vol}_{n-2}\left(F_{t}\right)
\end{aligned}
$$

for $|t|$ small enough. By Lemma 5.2, this expression is equal to zero. Therefore, $\operatorname{vol}(., c)$ is constant on the path-connected components of the space of representations $\mathcal{R}$. Hence the result.

\section{Proof of Theorem B}

Proposition 6.1. Let $\left\{M_{i}\right\}$ be a sequence of infinitely many, non-homeomorphic, closed oriented hyperbolic n-manifolds. Then, the sequence $\left\{h\left(M_{i}\right)\right\}$ of their simplicial heights is unbounded.

Proof. We follow the arguments developed in [BCG05, Théorème 6.6], which provide an alternate proof to a result of T. Soma [So98].

We argue by contradiction and assume that the simplicial height of the manifolds $M_{i}$ is bounded by an integer $N$, cf. Definition 2.2. Let $\Psi_{i}: P_{i} \rightarrow$ $M_{i}$ be a map from a finite simplicial complex $P_{i}$ with at most $N$ simplices which induces an epimorphism $\Psi_{i *}: H_{n}\left(P_{i}, \mathbb{Z}\right) \rightarrow H_{n}\left(M_{i}, \mathbb{Z}\right)$ between the $n$ th homology groups. Such maps exist by assumption since $M_{i}$ is aspherical, that is a $K\left(\pi_{1}\left(M_{i}\right), 1\right)$-space. For every integer $i$, choose a family of $n$-cycles $c_{i}^{1}, \ldots, c_{i}^{m_{i}}$ of $P_{i}$ inducing in homology a basis of the $\mathbb{Z}$-module $H_{n}\left(P_{i}, \mathbb{Z}\right)$. For every pair $i, j$ such that $P_{i}=P_{j}$ (notice that there are finitely many simplicial complexes $P_{i}$ ), we can further impose that $c_{i}^{k}=c_{j}^{k}$ for every $k=$ $1, \ldots, m_{i}\left(=m_{j}\right)$. Since the homomorphism $\Psi_{i *}: H_{n}\left(P_{i}, \mathbb{Z}\right) \rightarrow H_{n}\left(M_{i}, \mathbb{Z}\right)$ is nonzero, there is an $n$-cycle $c_{i}=c_{i}^{k_{i}}$ of $P_{i}$ such that $\Psi_{i *}\left[c_{i}\right]=d_{i}\left[M_{i}\right]$ where $d_{i}$ is a nonzero integer. The properties of the simplicial volume, $c f$. [Gr81], imply that

$$
\left\|\left[c_{i}\right]\right\| \geq\left\|\Psi_{i *}\left[c_{i}\right]\right\|=\left|d_{i}\right| \cdot\left\|M_{i}\right\| .
$$


Since $M_{i}$ is a closed hyperbolic manifold, its simplicial volume, which is proportional to its volume $c f$. [Gr81], is bounded away from zero, $c f$. [Th97]. Thus, the sequence $\left\{d_{i}\right\}$ is bounded (recall that $\left\|\left[c_{i}\right]\right\|$ takes finitely many values).

Let $\rho_{i}: \pi_{1}\left(P_{i}\right) \rightarrow \operatorname{Isom}\left(\mathbb{H}^{n}\right)$ be the representation induced by $\Psi_{i}$. By construction, the relation

$$
\operatorname{vol}\left(\rho_{i}, c_{i}\right)=d_{i} \operatorname{vol} M_{i}
$$

holds for every $i$. By Proposition 5.1, the volume of the closed hyperbolic manifolds $M_{i}$ takes finitely many values (recall that $d_{i}$ is nonzero and bounded). Therefore, there are finitely many closed hyperbolic manifolds $M_{i}, c f$. [Th97].

Remark 6.2. To cover the nonorientable case, one can use $\mathbb{Z}_{2}$ homology coefficients in the definition of the simplicial height, $c f$. Definition 2.2. However, the example of a nonorientable closed hyperbolic 3-manifold admitting maps of $\mathbb{Z}_{2}$-degree 1 onto infinitely many closed hyperbolic manifolds, $c f$. [BW96], [Ro95], shows that the conclusion of Proposition 6.1 fails in this case.

We can now conclude.

Proof of Theorem B. Combined with the inequality (1.4), Proposition 6.1 implies Theorem B in the orientable case. In the nonorientable case, we consider the orientable double Riemannian cover $\bar{M} \rightarrow M$. Since the quotient map from $\bar{M}$ to $M$ is a local isometry which induces an injective homomorphism between the fundamental groups, we have $\operatorname{sys}(\bar{M}) \geq \operatorname{sys}(M)$. On the other hand, $\operatorname{vol}(\bar{M})=2 \operatorname{vol}(M)$. Hence $\sigma(\bar{M}) \leq 2 \sigma(M)$. Now, since only finitely many hyperbolic $n$-manifolds have the same volume when $n \geq 3$, no subsequence of $\left\{\bar{M}_{i}\right\}$ is constant. By Theorem B (orientable case), the systolic volume of the $\bar{M}_{i}$ 's, and so of the $M_{i}$ 's, is unbounded. Hence the result.

Example 6.3 (Systolic volume of tubes). Let $\mathbb{T}^{2}$ be a flat 2-torus with systole $\ell$. For every prime class $c \in \pi_{1}\left(\mathbb{T}^{2}\right) \simeq \mathbb{Z}^{2}$, we can fill $\mathbb{T}^{2}$ into a solid 3 -torus $T_{c}^{3}$ such that the kernel of the $\pi_{1}$-homomorphism induced by the inclusion $\partial T_{c}^{3} \subset T_{c}^{3}$ is generated by $c$. For every prime $c \in \pi_{1}\left(\mathbb{T}^{2}\right)$, we can construct a metric $g$ on $T_{c}^{3}$ such that

(C1) $g$ agrees with the initial flat metric on $\partial T_{c}^{3}=\mathbb{T}^{2}$;

(C2) $\operatorname{sys}\left(\partial T_{c}^{3}, g\right)=\ell$.

Is it possible to do so while controlling the volume of the filling? More precisely, is there a constant $V_{0}$ such that, for every prime $c \in \pi_{1}\left(\mathbb{T}^{2}\right)$, there exists a metric $g$ on $\partial T_{c}^{3}=\mathbb{T}^{2}$ satisfying the conditions $(\mathrm{C} 1)$ and $(\mathrm{C} 2)$ with $\operatorname{vol}\left(\partial T_{c}^{3}, g\right) \leq V_{0}$ ? This question, which naturally arises when one tries to construct a counterexample to Theorem B (see below), has a negative answer: there is no such uniform upper bound on the volume of $\partial T_{c}^{3}$ (for a metric satisfying $(\mathrm{C} 1)$ and $(\mathrm{C} 2))$. Specifically, let $c_{n} \in \pi_{1}\left(\mathbb{T}^{2}\right)$ be a sequence 
of infinitely many distinct prime classes and $g_{n}$ be a sequence of metrics on $T_{c_{n}}^{3}$ satisfying $(\mathrm{C} 1)$ and $(\mathrm{C} 2)$, then $\operatorname{vol}\left(g_{n}\right) \rightarrow \infty$.

To prove this result, we argue by contradiction and consider a complete hyperbolic 3-manifold $M$ of finite volume with one cusp. Cut this cusp along a horosphere far enough and remove the noncompact convex component. One obtains a compact hyperbolic 3-manifold with boundary a flat torus $\mathbb{T}^{2}$ along which we glue a solid 3-torus $T_{c}^{3}$. These Dehn surgeries yield infinitely many, non-homeomorphic, closed 3-manifolds $M_{c}$ admitting a hyperbolic metric, $c f$. [Th97]. By construction, the systole of $M_{c}$ agrees with $\ell$ and the volume of $M_{c}$ is less than $\operatorname{vol}(M)+V$. Thus, the systolic volume of $M_{c}$ is bounded. Hence a contradiction with Theorem B.

\section{REFERENCES}

[Ba93] Babenko, I.: Asymptotic invariants of smooth manifolds. Russian Acad. Sci. Izv. Math. 41 (1993) 1-38.

[Ba95] Babenko, I.: Extremal problems in geometry, surgeries of manifolds and problems in group theory. Izv. Math. 59 (1995) 321-332.

[Ba04] Babenko, I.: Géométrie systolique des variétés de groupe fondamental $Z_{2}$, Sémin. Théor. Spectr. Géom. 22 (2004), 25-52.

[Ba06] Babenko, I.: Nature topologique des systoles unidimensionnelles. Enseign. Math. (2) 52 (2006), no. 1-2, 109-142.

[BB05] Babenko, I.; Balacheff, F.: Géométrie systolique des sommes connexes et des revêtements cycliques, Math. Ann. 333 (2005), no. 1, 157-180.

[BCIK06] Bangert, V; Croke, C.; Ivanov, S.; Katz, M.: Boundary case of equality in optimal Loewner-type inequalities, Trans. A.M.S. 359 (2007), no. 1, 1-7.

[BK03] Bangert, V.; Katz, M.: Stable systolic inequalities and cohomology products, Comm. Pure Appl. Math. 56 (2003), 979-997.

[BK04] Bangert, V; Katz, M.: An optimal Loewner-type systolic inequality and harmonic one-forms of constant norm. Comm. Anal. Geom. 12 (2004), 701-730.

[BCG05] Besson, G.; Courtois, G.; Gallot, S.: Inégalités de Milnor-Wood géométriques, Prépublication de l'Institut Fourier, no. 666 (2005), available at http://wwwfourier.ujf-grenoble.fr/cgi-bin/envoips.pl?PS=PREP/ps/t666

[BW96] Boileau, M.; Wang, S. C.: Non-zero degree maps and surface bundles over $S^{1}, J$. Diff. Geom. 43, (1996) 789-908.

[BGLM02] Burger, M.; Gelander, T.; Lubotzky, A.; Mozes, S.: Counting hyperbolic manifolds, Geom. Funct. Anal. 12 (2002), no. 6, 1161-1173.

[BS94] Buser, P.; Sarnak, P.: On the period matrix of a Riemann surface of large genus. With an appendix by J. H. Conway and N. J. A. Sloane. Invent. Math. 117 (1994), $27-56$.

[CK03] Croke, C.; Katz, M.: Universal volume bounds in Riemannian manifolds, Yau, S.-T. (ed.), Surveys in Differential Geometry VIII, Lectures on Geometry and Topology held in honor of Calabi, Lawson, Siu, and Uhlenbeck at Harvard University, May 3-5, 2002. Somerville, MA: International Press, 2003, pp. 109-137.

[Du99] Dunfield, N.: Cyclic surgery, degrees of maps of character curves, and volume rigidity for hyperbolic manifolds. Invent. Math. 136 (1999), no. 3, 623-657.

[Gr81] Gromov, M.: Volume and bounded cohomology. Publ. IHES. 56 (1981), 213-307.

[Gr83] Gromov, M.: Filling Riemannian manifolds. J. Differential Geom. 18 (1983), 1147. 
[Gr96] Gromov, M.: Systoles and intersystolic inequalities. In: Actes de la Table Ronde de Géométrie Différentielle (Luminy 1992), 291-362. Séminaires et Congrès, 1. Soc. Math. France, Institut Henri Poincaré, Paris 1996.

[Gr99] Gromov, M.: Metric structures for Riemannian and non-Riemannian spaces, Progress in Mathematics 152. Birkhäuser, Boston 1999.

[Gu] Guth L.: Volumes of balls in large Riemannian manifolds, preprint available at arXiv:math.DG/0610212

[IK04] Ivanov, S.; Katz, M.: Generalized degree and optimal Loewner-type inequalities, Israel J. Math. 141 (2004), 221-233.

[KR] Katz, M.; Rudyak, Yu.: Bounding volume by systoles of 3-manifolds, preprint available at arXiv:math.DG/0504008.

[KS05] Katz, M.; Sabourau, S.: Entropy of systolically extremal surfaces and asymptotic bounds, Ergo. Th. Dynam. Sys. 25 (2005), no. 4, 1209-1220.

[KSV05] Katz, M.; Schaps, M.; Vishne, U.: Logarithmic growth of systole of arithmetic Riemann surfaces along congruence subgroups, preprint available at arXiv:math.DG/0505007

[Ma77] Massey, W.: Algebraic topology: an introduction, Graduate Texts in Mathematics, Vol. 56, Springer-Verlag, 1977

[Mi94] Milnor, J.: The Schläfli differential inequality, Collected papers, Vol. 1, Geometry, Publish or Perish, Inc., Houston, TX, 1994.

[Re96] Reznikov, A.: Rationality of secondary classes, J. Differential Geom. 43 (1996), no. 3, 674-692.

[Ro95] Rong, Y.: Degree one maps of Seifert manifolds and a note on Seifert volume, Topology Appl. 64 (1995), no. 2, 191-200.

[RS] Rudyak, Yu. ; Sabourau, S.: Systolic invariants of groups and 2-complexes via Grushko decomposition, preprint available at arXiv:math.DG/0609379.

[Sa06] Sabourau, S.: Systolic volume and minimal entropy of aspherical manifolds, J. Differential Geom. 74 (2006), no. 1, 155-176.

[So98] Soma, T.: Non-zero degree maps to hyperbolic 3-manifolds, J. Differential Geom. 49 (1998), no. 3, 517-546.

[Sp66] Spanier, E.: Algebraic topology, Springer-Verlag, New-York, 1966.

[Sw75] Swan, R.: Thom's theory of differential forms on simplicial sets, Topology 14 (1975), no. 3, 271-273.

[Th97] Thurston, W.: Three-dimensional geometry and topology, Vol. 1, Ed. Silvio Levy. Princeton Mathematical Series, 35, Princeton Univ. Press, Princeton, 1997.

[Wa72] Wang, H. C.: Topics on totally discontinuous groups, Symmetric spaces, Pure and Appl. Math., Vol. 8, pp. 459-487, Dekker, New York, 1972.

Laboratoire de Mathématiques et Physique Théorique, Université de Tours, Parc de Grandmont, 37400 Tours, France

E-mail address: sabourau@lmpt.math.univ-tours.fr 